\title{
Flexible magnifying endoscopy with narrow band imaging versus colposcopy for diagnosing uterine cervical neoplasms: a multicenter, prospective, non-randomized, paired comparison study
}

\author{
Hideki Kobara ${ }^{1}$, Kunihisa Uchita ${ }^{2}$, Noriya Uedo $^{3}$, Jun Kunikata ${ }^{1}$, Kenji Yorita ${ }^{2}$, Naoya \\ Tada $^{1}$, Noriko Nishiyama ${ }^{1}$, Yuriko Shigehisa ${ }^{2}$, Chihiro Kuroiwa ${ }^{2}$, Noriko Matsuura ${ }^{3}$, Yohei \\ Takahashi $^{2}$, Yuka Kai ${ }^{2}$, Uiko Hanaoka ${ }^{1}$, Yumiko Kiyohara ${ }^{3}$, Shoji Kamiura ${ }^{3}$, Kenji \\ Kanenishi $^{1}$, Tsutomu Masaki ${ }^{1}$, and Koki Hirano ${ }^{2}$ \\ ${ }^{1}$ Kagawa University Faculty of Medicine Graduate School of Medicine \\ ${ }^{2}$ Kochi Sekijuji Byoin \\ ${ }^{3}$ Osaka International Cancer Institute
}

January 3, 2021

\begin{abstract}
Objective To investigate the detection ability of flexible magnifying endoscopy with narrow band imaging (ME-NBI) for cervical intraepithelial neoplasia grade two or worse (CIN2+) compared with colposcopy. Design Multicenter, prospective, nonrandomized, paired comparison study. Setting Three Japanese medical centers. Population Japanese women. Methods Eligible patients had positive PAP smear test results, suspicious high-grade CIN in previous colposcopy, or definitive CIN3 diagnosed previously. A gastrointestinal endoscopist examined the cervix using ME-NBI in an endoscopy room and, subsequently, a gynecologist blinded to the ME-NBI findings performed colposcopy in a different room. CIN2+ locations were documented in a scheme immediately after each examination. Punch biopsy samples were obtained from all areas diagnosed as CIN2+ with both methods and from one normal area. The reference standard was the presence of at least one histological diagnosis of CIN2+ among all biopsy specimens. Main outcome measures The primary outcome was the detection sensitivity of patients with CIN2+, comparing ME-NBI and colposcopy. Results We enrolled 88 patients. The detection sensitivity for patients with CIN2+ was not statistically different between the two methods (both: 79\%, 95\% CI: 66\%-88\%). For diagnosing CIN2+, MENBI tended to show a higher sensitivity than colposcopy ( $69 \%$ vs. $58 \%$, respectively), while its specificity tended to be lower vs. colposcopy ( $55 \%$ vs. $70 \%$, respectively). Patients reported significantly less discomfort and embarrassment with ME-NBI vs. colposcopy. Conclusion ME-NBI showed comparable sensitivity to colposcopy for detecting CIN2+ lesions, and ME-NBI was more patient-acceptable.
\end{abstract}

\section{Hosted file}

F*Main text BJOG.pdf available at https://authorea.com/users/363139/articles/502279-flexiblemagnifying-endoscopy-with-narrow-band-imaging-versus-colposcopy-for-diagnosing-uterinecervical-neoplasms-a-multicenter-prospective-non-randomized-paired-comparison-study 


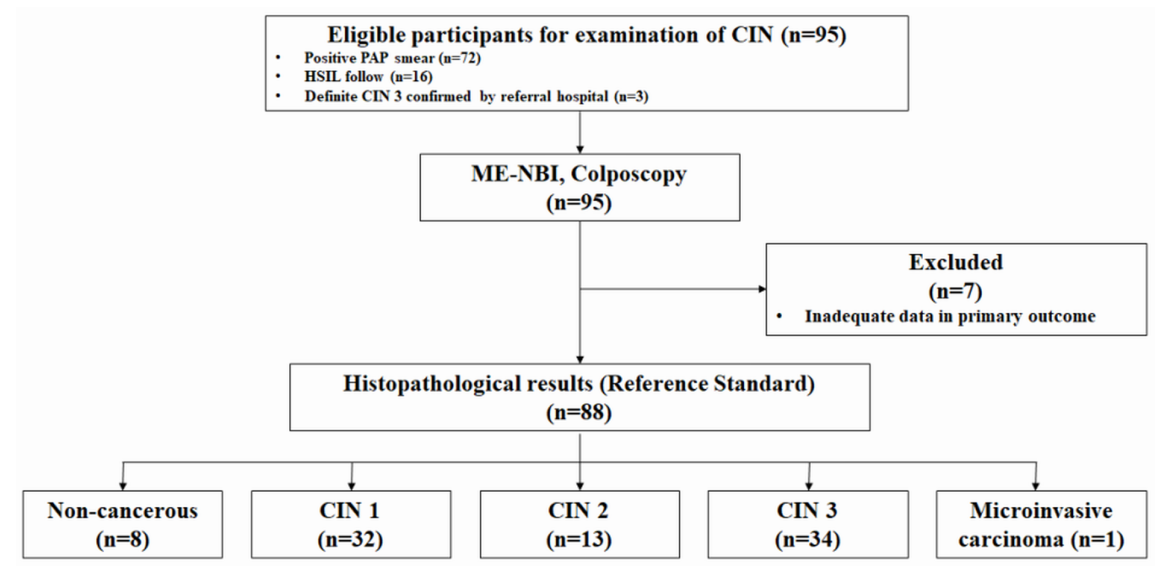

